\title{
Kohn-Sham potential for a strongly correlated finite system with fractional occupancy
}

\author{
A. Beníter ${ }^{*}$ and C. R. Proett田 \\ Centro Atómico Bariloche and Instituto Balseiro, \\ Comisión Nacional de Energía Atómica, 8400 Bariloche, Argentina
}

(Dated: March 18, 2022)

\begin{abstract}
Using a simplified one-dimensional model of a diatomic molecule, the associated interacting density and corresponding Kohn-Sham potential have been obtained analytically for all fractional molecule occupancies $N$ between 0 and 2. For the homonuclear case, and in the dissociation limit, the exact Kohn-Sham potential builds a barrier at the midpoint between the two atoms, whose strength increases linearly with $N$, with $1<N \leq 2$. In the heteronuclear case, the disociating KS potential besides the barrier also exhibits a plateau around the atom with the higher ionization potential, whose size (but not its strength) depends on $N$. An anomalous zero-order scaling of the Kohn-Sham potential with regards to the strength of the electron-electron repulsion is clearly displayed by our model; without this property both the unusual barrier and plateau features will be absent.
\end{abstract}

\section{INTRODUCTION}

Ground-state density-functional theory (DFT) maps the interacting electronic problem into an effective noninteracting system, that shares with the real system the ground-state density and energy [1, 2]. In the KohnSham (KS) formulation of DFT [3], the electrons are acted on by an effective single-particle, multiplicative potential, the KS potential. All the complicated many-body effects of the real system are fully included in this effective potential, whose crucial role is to do whatever is needed to reproduce the density and total energy of the real interacting system. The aim of the present work is to explore some of the unusual features of the exact KS potential, in particular its behavior when the system under study has a non-integer (fractional) number of electrons.

There are several reasons why the analysis of systems with fractional charges within the DFT framework may be of some interest. A good example is the behavior of the molecule $\mathrm{H}_{2}^{+}$, when stretched by increasing the distance between the two protons beyond the equilibrium separation. At the dissociation limit, and without any symmetry breaking, $\mathrm{H}_{2}^{+}$correctly splits in two identical fragments or open subsystems, each one consisting of a proton and half of one electron: $H^{+0.5} \cdots H^{+0.5}$. Most of the local or semilocal energy functionals of common use in DFT behave poorly in this limit of fractional occupancies, yielding energies far below the proper binding energy of 1 Hartree, due to the tendency of approximate functionals to spread out the electron density artificially. In practical calculations, the problem may be "solved" by breaking the spatial symmetry and localizing the electron on one of the two protons. Proceeding this way, the resulting binding energy of the $\mathrm{H}_{2}^{+}$molecule at its dissociation limit is reasonable, but the associated density is not correct. In exact DFT, on the other side, the prob-

\footnotetext{
* Present address: Catalan Institute of Nanoscience and Nanotechnology (ICN2), Campus UAB, Barcelona 08193, Spain

† Electronic address: proetto@cab.cnea.gov.ar
}

lem is solved by the condition that the energy of the two hydrogen-like fragments must be the same either if the electron is localized in one of the protons, or if there is half of one electron at each proton [4]. The same happens with all radical symmetric molecules $A_{2}^{+}$at infinite bond length. This failure has been denoted the "manyelectron self-interaction error" [5, 6] or "delocalization error" 7] of semilocal functionals, and happens when some occupied KS orbitals share an electron between two open subsystems, equivalent to having in each subsystem a non-integer occupation number. A related situation occurs with many asymmetric molecules $A B$ that with approximate functionals dissociate not to neutral atoms $A$ and $B$ but improperly to fractionally charged fragments $A^{+q} \cdots B^{-q}$ [5]. Also, long-range charge transfers are usually overestimated [8], and the energy barriers that control the reaction rates in chemical reactions are underestimated or even absent 9. All these problematic issues of current DFT may be related to specific missing features of the KS potential resulting from semilocal functionals.

From a rigorous point of view, the extension of the ground-state DFT formalism to the case of systems whose density integrates to a fractional number was made in a seminal work by Perdew et al. [10. They proved, by introducing a zero-temperature ensemble DFT formalism, that the total energy is a piecewise linear function of the electron number between two adjacent integers. In turn, this leads to the theoretical prediction of a discontinuity of the KS potential as the electron number passes through an integer. This abrupt jump of the exact KS potential, missed in all local or semilocal approximations, is on the other side the crucial ingredient that explains the severe underestimation of the fundamental band-gap of insulators and semiconductors [11, 12. All these issues have been discussed in the influential review of Ref. [13]; for a more updated review, see Ref. [14.

The aim of this work is to illustrate some of the nonintuitive features of the KS potential through the use of a simple but strongly correlated one-dimensional model of the hydrogen molecule, for which the exact interact- 
ing density is available for any electron number between 0 and 2. By a reverse-engineering procedure, the $N$ dependent exact KS potential is then obtained from the $N$-dependent density. The simplicity of our model allow us to prove unambiguously an anomalous scaling of the correlation potential, with regard to the strength of the electron-electron repulsion. The present work is organized as follows: in Section II we introduce the model and explain the method we use for finding its solutions; in Section III we provide the main numerical and analytical results, while Section IV is devoted to the Conclusions.

\section{MODEL AND METHOD OF SOLUTION}

The bottleneck of our reverse-engineering method is in finding the solution (in principle exact) of a $N=2$ closed shell diatomic molecule. As this is not easily available, and we are more interested in understanding the physics behind the KS potential than in describing a real tridimensional molecule, we will simplify the model. Following Ref. 15, we will use the following one-dimensional mimic of a diatomic molecule (in atomic units (a.u.))

$$
\begin{array}{r}
{\left[-\frac{1}{2}\left(\frac{\partial^{2}}{\partial x_{1}^{2}}+\frac{\partial^{2}}{\partial x_{2}^{2}}\right)+v_{\text {ext }}\left(x_{1}\right)+v_{\text {ext }}\left(x_{2}\right)\right.} \\
\left.+v_{\text {int }}\left(\left|x_{1}-x_{2}\right|\right)\right] \Psi^{\gamma}\left(x_{1}, x_{2}\right)=E(2) \Psi^{\gamma}\left(x_{1}, x_{2}\right),
\end{array}
$$

with

$$
v_{\text {ext }}(x)=-v[\lambda \delta(x+d / 2)+\delta(x-d / 2)],
$$

and

$$
v_{\text {int }}\left(\left|x-x^{\prime}\right|\right)=\gamma \delta\left(x-x^{\prime}\right) .
$$

As we will see, this simplified model preserves all the main physical ingredients of the real three-dimensional molecule with the long-range Coulomb interactions. Here, $v$ and $\lambda$ are both positive; $\lambda=1(\neq 1)$ corresponds to the homonuclear (heteronuclear) diatomic molecule. Since a one-dimensional attractive delta-potential has only one bound state [16], the two attractive delta potentials in $v_{\text {ext }}(x)$ provides the two-dimensional boundstates basis needed for the forthcoming considerations. $v_{\text {ext }}(x)$ represents in our model the attractive Coulomb potentials from the two protons of the real hydrogen molecule. Eq. (3) represents the repulsive interaction $(\gamma \geq 0)$ between the two electrons in the molecule, expressed here by a short-range (contact) interaction. The non-interacting limit of Eq. (1) is obtained for $\gamma=0$, while $\gamma \rightarrow \infty$ drives the "molecule" towards the stronglyinteracting limit. The value of the ratio $v / \gamma$ moves the system from the weakly interacting regime $(v / \gamma \gg 1)$ towards the strongly interacting regime $(v / \gamma \ll 1)$. And the nice point is that the ground-state $\Psi^{\gamma}\left(x_{1}, x_{2}\right)$ and $E(2)$ may be found analytically for $\gamma \rightarrow \infty$, by appealing to the boson-fermion mapping [17. More precisely,

$$
\Psi^{\gamma \rightarrow \infty}\left(x_{1}, x_{2}\right)=\left|\phi_{+}\left(x_{1}\right) \phi_{-}\left(x_{2}\right)-\phi_{+}\left(x_{2}\right) \phi_{-}\left(x_{1}\right)\right|,
$$

and $E(2)=\varepsilon_{+}+\varepsilon_{-}$. In equation above, the symbol $|\ldots|$ represents the absolute value, and $\phi_{ \pm}(x)$ and $\varepsilon_{ \pm}$are the normalized eigenfunctions and eigenvalues of the following single-particle Schrödinger equation,

$$
\left[-\frac{1}{2} \frac{\partial^{2}}{\partial x^{2}}+v_{\text {ext }}(x)\right] \phi_{ \pm}(x)=\varepsilon_{ \pm} \phi_{ \pm}(x) .
$$

From Eqs. (5) and (2) one obtains

$$
\phi_{ \pm}(x)=A_{ \pm}\left(e^{-\alpha_{ \pm}|x+d / 2|}+f_{ \pm} e^{-\alpha_{ \pm}|x-d / 2|}\right)
$$

with

$$
\begin{gathered}
A_{ \pm}^{2}=\frac{\alpha_{ \pm}}{1+f_{ \pm}^{2}+2 f_{ \pm} e^{-d \alpha_{ \pm}}\left(1+d \alpha_{ \pm}\right)} \\
\alpha_{ \pm}=\frac{v(1+\lambda)}{2} \pm \frac{v}{2}\left[(1-\lambda)^{2}+4 \lambda e^{-2 d \alpha_{ \pm}}\right]^{1 / 2} \\
f_{ \pm}=\frac{v e^{-d \alpha_{ \pm}}}{\alpha_{ \pm}-v}
\end{gathered}
$$

and $\varepsilon_{ \pm}=-\alpha_{ \pm}^{2} / 2 ;$ since $\alpha_{+} \geq \alpha_{-}, \varepsilon_{+} \leq \varepsilon_{-}$. The density of the two-electron system is defined by

$$
\rho_{2}(x):=\int\left[\Psi^{\gamma \rightarrow \infty}\left(x, x^{\prime}\right)\right]^{2} d x^{\prime}=\phi_{+}^{2}(x)+\phi_{-}^{2}(x),
$$

which should be contrasted with the non-interacting twoelectron density $\rho_{2}^{0}(x)=2 \phi_{+}^{2}(x)$.

Following the prescription of zero-temperature ensemble DFT [1, 2, the ground-state density for the diatomic molecule with $N$ electrons, with $0 \leq N \leq 2$, is given by

$\rho_{N}(x)= \begin{cases}N \rho_{1}(x) & \text { if } 0 \leq N \leq 1, \\ (2-N) \rho_{1}(x)+(N-1) \rho_{2}(x) & \text { if } 1 \leq N \leq 2,\end{cases}$

with $\rho_{2}(x)$ defined in Eq. 10, , and $\rho_{1}(x)=\phi_{+}^{2}(x)$ [18]. Replacing we obtain that

$$
\rho_{N}(x)= \begin{cases}N \phi_{+}^{2}(x) & \text { if } 0 \leq N \leq 1, \\ \phi_{+}^{2}(x)+(N-1) \phi_{-}^{2}(x) & \text { if } 1 \leq N \leq 2 .\end{cases}
$$

This result for the interacting density should be contrasted with the $N$-dependent non-interacting density $\rho_{N}^{0}(x)=N \phi_{+}^{2}(x)$, for $0 \leq N \leq 2$. Both $\rho_{N}(x)$ and $\rho_{N}^{0}(x)$ are identical for $0 \leq N \leq 1$, as it should be. However, for $N>1$, while $\rho_{N}^{0}(\bar{x})$ continues with the progressive occupancy of the single-particle ground-state orbital $\phi_{+}(x), \rho_{N}(x)$ places the fractional occupancy beyond unity fully in the first-excited orbital $\phi_{-}(x)$. This striking difference between both densities is displayed in Fig. 1 for the homonuclear case $(\lambda=1)$, and in Fig. 2 for the heteronuclear case $(\lambda \neq 1)$. 


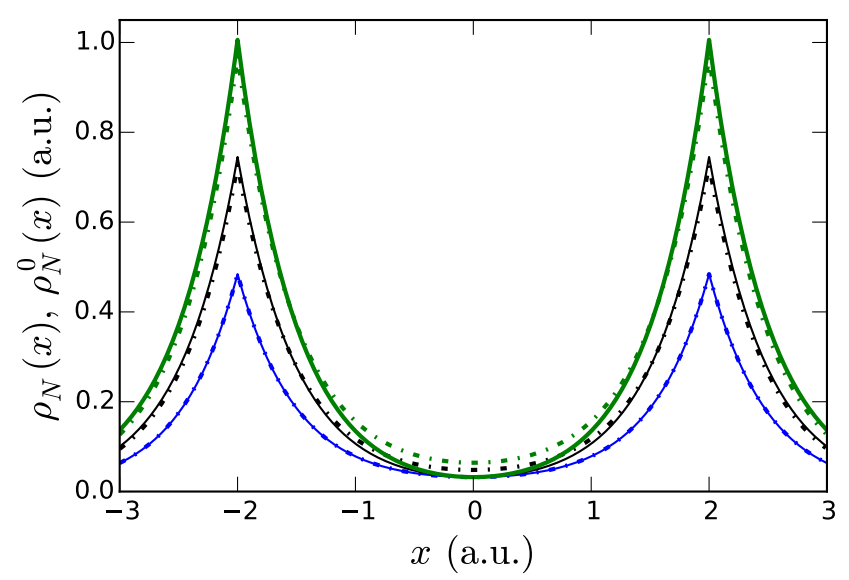

FIG. 1. $\rho_{N}^{0}(x)$ (dotted lines) and $\rho_{N}(x)$ (full lines) for the homonuclear case, and $N=1$ (blue), 1.5 (black), 2 (green). $v=\lambda=1$, and $d=4$. For $N=1$ both densities coincide.

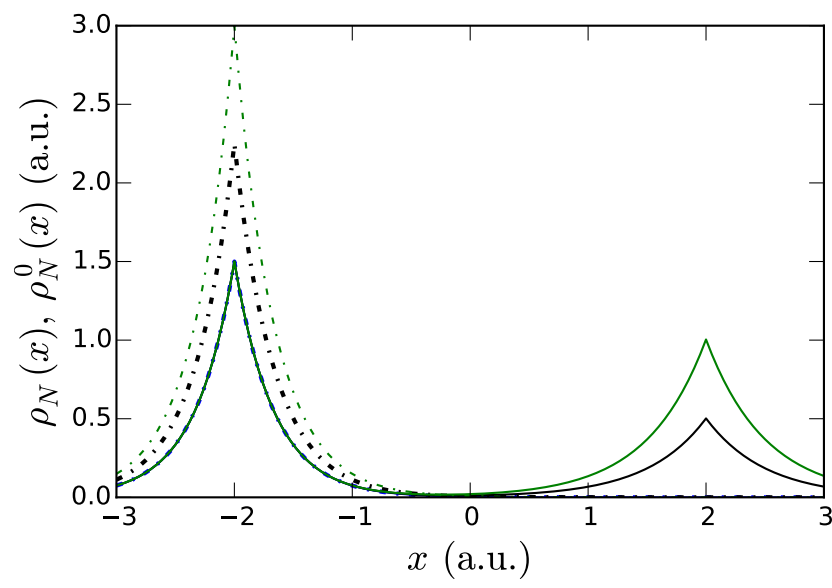

FIG. 2. $\rho_{N}^{0}(x)$ (dotted lines) and $\rho_{N}(x)$ (full lines) for the heteronuclear case, and $N=1$ (blue), 1.5 (black), 2 (green). $v=1, \lambda=1.5$, and $d=4$. For $N=1$ both densities coincide.

Having $\rho_{N}(x)$, the KS potential is obtained by reverse engineering from the expression

$$
v_{\mathrm{KS}}^{N}(x)=\frac{1}{2 \sqrt{\rho_{N}(x)}} \frac{\partial^{2} \sqrt{\rho_{N}(x)}}{\partial x^{2}}+C,
$$

with $C$ being a constant to be fixed later. While Eq. (13) is self-evident in the KS framework for $N=1$ and $N=2$, its applicability in the full range $0 \leq N \leq 2$ has been discussed and validated in Ref. 19. Replacing the $N$ dependent density $\rho_{N}(x)$ in Eq. 13), it yields

$$
v_{\mathrm{KS}}^{N}(x)= \begin{cases}v_{\mathrm{ext}}(x)+\Delta v_{\mathrm{KS}}^{<}(x) & \text { if } 0 \leq N \leq 1, \\ v_{\mathrm{ext}}(x)+\Delta v_{\mathrm{KS}}^{>}(x) & \text { if } 1<N \leq 2,\end{cases}
$$

where $\Delta v_{\mathrm{KS}}^{<}(x)=\varepsilon_{+}+C^{<}, \Delta v_{\mathrm{KS}}^{>}(x)=v_{\mathrm{C}}^{\text {barr }}(x)+$ $v_{\mathrm{C}}^{\mathrm{step}}(x)+C^{>}$, and

$$
\begin{gathered}
v_{\mathrm{C}}^{\mathrm{barr}}(x)=\frac{(N-1)\left[\phi_{+}^{\prime}(x) \phi_{-}(x)-\phi_{-}^{\prime}(x) \phi_{+}(x)\right]^{2}}{2\left[\rho_{N}^{>}(x)\right]^{2}}, \\
v_{\mathrm{C}}^{\text {step }}(x)=-\frac{\varepsilon_{+} \phi_{+}^{2}(x)+(N-1) \varepsilon_{-} \phi_{-}^{2}(x)}{\rho_{N}^{>}(x)}
\end{gathered}
$$

with $\rho_{N}^{>}(x)$ equal to $\rho_{N}(x)$ for $N \geq 1$, and primes denoting derivate with respect to the coordinate $x$. For $N=2, \Delta v_{\mathrm{KS}}^{>}(x)$ reduces to the expression obtained in Ref. 15] for the same model. Choosing $C^{<}=$ $-\varepsilon_{+}$, we obtain that $\Delta_{\mathrm{KS}}^{<}(x) \equiv 0$. In the asymptotic limit $|x| \gg d, \phi_{+}(x) / \phi_{-}(x) \rightarrow 0, v_{\mathrm{C}}^{\mathrm{barr}}(x)$ goes to zero, while $v_{\mathrm{C}}^{\text {step }}(x)$ approaches $-\varepsilon_{-}$; as a consequence $\Delta_{\mathrm{KS}}^{>}(x \gg d) \rightarrow-\varepsilon_{-}+C^{>}$. Choosing $C^{>}=$ $\varepsilon_{-}, \quad \Delta_{\mathrm{KS}}^{>}(|x| \gg d) \rightarrow 0 . \quad \Delta v_{\mathrm{KS}}^{>}(x)$ has the following interesting property: $\lim _{|x| \rightarrow \infty} \Delta v_{\mathrm{KS}}^{>}(x) \rightarrow 0$, but $\lim _{|x| \rightarrow \infty} \lim _{N \rightarrow 1^{+}} \Delta v_{\mathrm{KS}}^{>}(x) \rightarrow \varepsilon_{-}-\varepsilon_{+}=I(1)-A(1)>$ 0 [10. Here we have defined $I(N)=E(N-1)-E(N)$ and $A(N)=I(N+1)=E(N)-E(N+1)$ as the ionization potential and electronic affinity, respectively, of the $N$-electron molecule. This is precisely the discontinuity of the $N$-dependent KS potential addressed above, when crossing integer values of $N(N=1$ in this case). Using a different model with an external harmonic confinement, Ref. 20 analyzes the discontinuity for the case $N=1$, while in Ref. 21] the discontinuity has been analyzed for several real atoms, for other values of $N$.

The case $N=2$ of present model for the diatomic molecule has been generalized in Ref. [15], replacing the delta-function potentials in Eqs. (2) and (3) by softCoulomb potentials of the type $1 / \cosh ^{2}(x)$. The results between the two models are quite similar, particularly in the dissociation limit where the respective KS potentials become essentially identical.

\section{RESULTS AND DISCUSSIONS}

\section{A. Homonuclear case}

For $\lambda=1$, the eigenvalue equation simplifies to $\alpha_{ \pm}=v\left(1 \pm e^{-d \alpha_{ \pm}}\right)$, and this leads to $f_{ \pm}= \pm 1$, and $A_{ \pm}^{\overline{2}}=\left(\alpha_{ \pm} / 2\right) /\left[1 \pm e^{-d \alpha_{ \pm}}\left(1+d \alpha_{ \pm}\right)\right] . \quad \phi_{+}(x)$ becomes the symmetric "bonding" solution, while $\phi_{-}(x)$ becomes the antisymmetric "antibonding" solution. In the dissociation limit, defined as $v d \gg 1, \alpha_{+} \sim \alpha_{-} \sim v$, and since $\varepsilon_{-} \sim \varepsilon_{+} \sim-v^{2} / 2$, the "step" contribution to $\Delta v_{\mathrm{KS}}^{>}(x)$ becomes a constant that cancels with $C^{>}\left(=\varepsilon_{-}\right)$. For the "barrier" contribution one obtains that if $-d / 2<x<d / 2$,

$$
v_{\mathrm{C}}^{\mathrm{barr}}(x, v d \gg 1) \simeq \frac{4 I(N-1)}{[2-N+N \cosh (2 \sqrt{2 I} x)]^{2}},
$$


with $I=v^{2} / 2$ being the ionization potential of any of the two identical fragments. Clearly $v_{\mathrm{C}}^{\text {barr }}(x)$ vanishes for $N=1$ and has the largest value for $N=2$, while as a function of the coordinate $x$ it has the shape of a barrier centered at $x=0$, of height $I(N-1)$. We display in Fig. 3 a drawing of $v_{\mathrm{C}}^{\text {barr }}(x)$ for two typical situations, using the expression in Eq. (15).

The physics behind this barrier in the KS potential is best understood by writing the interacting density for $1 \leq N \leq 2$ as

$$
\rho_{N}^{>}(x)=\rho_{N}^{0}(x)+(N-1)\left[\phi_{-}^{2}(x)-\phi_{+}^{2}(x)\right] .
$$

As may be appreciated from Fig. 1, the difference $\phi_{-}^{2}(x)-$ $\phi_{+}^{2}(x)$ is positive for $x \sim \pm d / 2$, but negative in the "bonding" region $x \sim 0$. Considering both facts, one concludes that the main difference between $\rho_{N}^{0}(x)$ and $\rho_{N}(x)$ is that the electronic charge (even when fractional) tends to be more localized around the "atoms" in the latter, as a way of diminishing the repulsive interaction. And the only way that the KS effective one-body potential has to provide a ground-state density equal to $\rho_{N}(x)$ is through the building of a barrier around the "bonding" region, as given by Eq. (17). It is interesting to note that once the dissociation limit $v d \gg 1$ sets in, $v_{\mathrm{C}}^{\mathrm{barr}}(x, v d \gg 1)$ is independent on the distance $d$ between the two atoms. The size of the barrier, on the other side, depends crucially on an atomic property of the two separated fragments, the ionization potential $I$, and also on the number of electrons in the molecule.

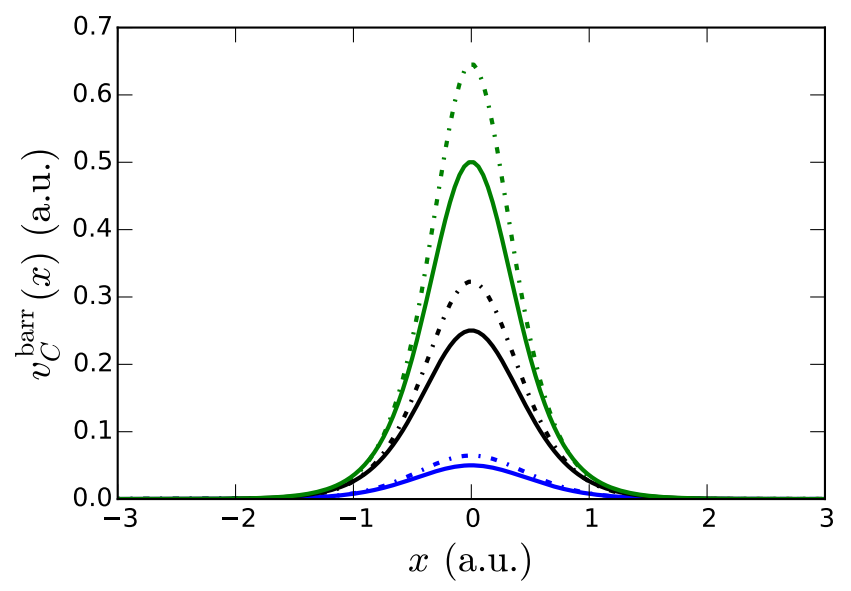

FIG. 3. $v_{\mathrm{C}}^{\text {barr }}(x)$ for $N=1.1$ (blue), 1.5 (black), 2 (green). $v=\lambda=1$, and $d=4$ (dotted lines) and 10 (full lines).

From Eq. 17) one obtains that $v_{\mathrm{C}}^{\mathrm{barr}}(0, v d \gg 1)=$ $I(N-1) \simeq 0.05,0.25$, and 0.50 for $N=1.1,1.5$, and 2 , respectively, and for the parameters in Fig. 3. This confirms that for $d=10, v_{\mathrm{C}}^{\text {barr }}(x)$ is fully in the dissociation limit, but not when $d=4$. When $v d \gg 1$, the electronic density in the bonding region is exponentially small $\left(\sim e^{-d v}\right)$, and then the only role of the barrier consists in maintain the difference $\phi_{-}^{2}(x)-\phi_{+}^{2}(x)$ positive for $x \sim \pm d / 2$. Its second role, depressing the density in the interatomic region becomes irrelevant in the dissociation limit, since as discussed above the density is exponentially small there. As a consequence, in the dissociation limit the barrier becomes essentially an atomic property, independent of the distance between the fragments, and with an "intrinsic" height of $I(N-1)$. When the two atoms become closer $(d=4$ in Fig. 3$)$, the height of the barrier increases beyond its dissociation limit, since it becomes more difficult to isolate the electronic charge of the two approaching fragments.

Returning to Eq. (12), it is seen that in the dissociation limit it can be written as

$$
\begin{aligned}
\rho_{N}^{>}(x, v d \gg 1) & \sim \frac{N}{2}\left[\rho_{L}(x)+\rho_{R}(x)\right] \\
& +(2-N) \sqrt{\rho_{L}(x)} \sqrt{\rho_{R}(x)}
\end{aligned}
$$

with $\rho_{L}(x)=\sqrt{2 I_{L}} e^{-2 \sqrt{2 I_{L}}|x+d / 2|}, \quad \rho_{R}(x)=$ $\sqrt{2 I_{R}} e^{-2 \sqrt{2 I_{R}}|x-d / 2|}$ being the normalized densities associated to the left and right fragments, respectively. In the approximation of Eq. (19), $I_{L} \simeq I_{R} \simeq I$. It shows that for $1 \leq N<2$, the interacting density is not the plain sum of the density of the left and right fragments. Only for $N=2, \rho_{2}(x, v d \gg 1) \sim \rho_{L}(x)+\rho_{R}(x)$, as found in Ref. [15]. Note that if, erroneously, one would approximate $\rho_{N}^{>}(x, v d \gg 1)$ by the sum of the two atomic densities, the resulting $v_{\mathrm{KS}}^{N}(x)$ would be independent of $N$ [22], contrary to the rigorous result for $v_{\mathrm{C}}^{\mathrm{barr}}(x, v d \gg 1)$ in Eq. (17). In other words, when electronic systems with fractional charges are involved, the hallmark of the dissociating limit is not always the fact that the density can be written as the sum of the isolated or atomic densities.

For $N=2$, the appearance of a potential barrier at the midpoint between the two protons in the $\mathrm{H}_{2}$ molecule has been discussed long ago, in works by Baerends and coworkers [23, 24]. What we have denoted here as $v_{\mathrm{C}}^{\text {barr }}(x)$ is what these authors denoted as $v_{\text {kin }}(\mathbf{r})$. Since the definition of this "kinetic" potential is in terms of a conditional probability, which in turn is defined only for integer occupancies (it will be $N=2$ in our case), the latter may be considered as a particular case of the former.

\section{B. Heteronuclear case}

As can be appreciated from Fig. 2, the evolution of $\rho_{N}^{>}(x)$ is quite different from the $\rho_{N}^{>}(x)$ for the homonuclear case displayed in Fig. 1, while also exists marked differences between the heteronuclear densities $\rho_{N}^{>}(x)$ and $\rho_{N}^{0}(x)$. The differences are easily seen in the dissociation limit $e^{-v d} \ll|1-\lambda|$; expanding the square root in Eq. (8), it yields

$$
\alpha_{ \pm} \simeq \frac{v(1+\lambda)}{2} \pm \frac{v|1-\lambda|}{2}\left[1+\frac{2 \lambda e^{-2 d \alpha_{ \pm}}}{(1-\lambda)^{2}}\right]
$$


To proceed, let us choose that $\lambda>1$. One obtains then that $\alpha_{+}(\lambda>1) \sim v \lambda, \alpha_{-}(\lambda>1) \sim v-v \lambda e^{-2 v d} /(\lambda-1)$, $f_{+}(\lambda>1) \sim e^{-v \lambda d} /(\lambda-1) \ll 1$, and $f_{-}(\lambda>1) \sim$ $(\lambda-1) e^{v d} / \lambda \gg 1$. Returning to Eq. (6), if $f_{+}(\lambda>1) \ll 1$, this implies that in the dissociating limit $\phi_{+}(x)$ essentially corresponds to $\phi_{L}(x)\left(=\sqrt{\rho_{L}(x)}\right)$, while $\phi_{-}(x)$ is essentially equal to $\phi_{R}(x)\left(=\sqrt{\rho_{R}(x)}\right)$. This already explains the results in Fig. 2: a) since $\rho_{N}^{0}(x)=$ $N \phi_{+}^{2}(x) \sim N \phi_{L}^{2}(x)$, increasing $N$ the density of the left-well increases, while the right-well remains essentially empty; b) since $\rho_{N}^{>}(x)=\phi_{+}^{2}(x)+(N-1) \phi_{-}^{2}(x) \simeq$ $\phi_{L}^{2}(x)+(N-1) \phi_{R}^{2}(x)$, as $N$ increases beyond unity now the density at the right-well is the one that increases, while the density at the left-well remains essentially constant, and equal to its value at $N=1 ;$ c) since $\alpha_{+}>\alpha_{-}$, the decay of the density around $x=-d / 2$ is faster than the decay of the density around $x=d / 2$. Choosing instead $\lambda<1$, the roles of $\phi_{L}(x)$ and $\phi_{R}(x)$ are exchanged, resulting in an interacting density, for instance, given by $\rho_{N}^{>}(x)=\phi_{R}^{2}(x)+(N-1) \phi_{L}^{2}(x)$.

This is the way that the interacting system finds to minimize the repulsive contact interaction: for $0 \leq N \leq$ 1 we have a non-interacting system and all the charge goes to the single-particle orbital with the lowest energy; for $1<N \leq 2$ the interacting system locates all the extra charge in the opposite well.

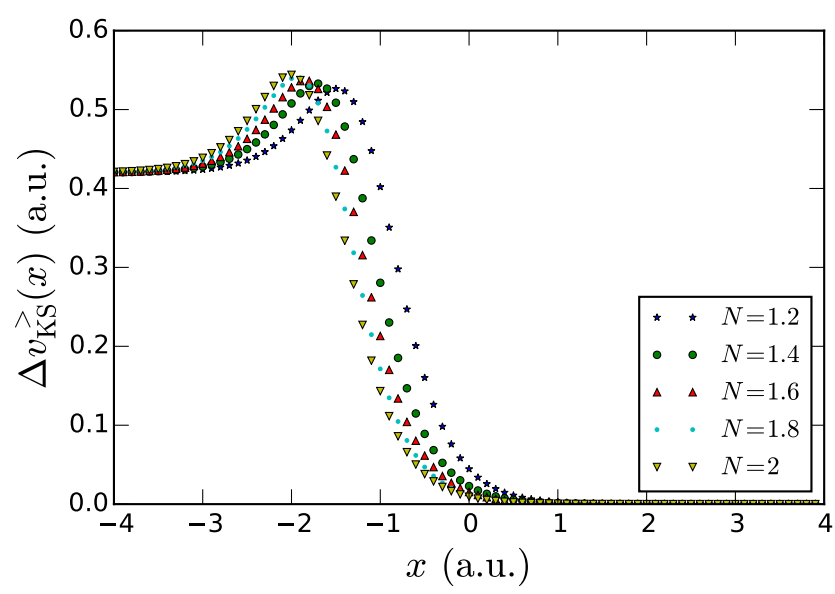

FIG. 4. $\Delta v_{\mathrm{KS}}^{>}(x)$ for $N=1.2,1.4,1.6,1.8,2 . v=0.4, \lambda=2.5$, and $d=10$.

We display in Fig. $4 \Delta v_{\mathrm{KS}}^{>}(x)$, for $d$ large enough such that the molecule is in the dissociation limit. The mapping $\phi_{+}(x) \rightarrow \phi_{L}(x)\left(\phi_{R}(x)\right)$ and $\phi_{-}(x) \rightarrow \phi_{R}(x)$ $\left(\phi_{L}(x)\right)$ for $\lambda>1(\lambda<1)$ in the dissociation limit provides us with alternative expressions for $v_{\mathrm{C}}^{\mathrm{barr}}(x, v d \gg 1)$ and $v_{\mathrm{C}}^{\text {step }}(x, v d \gg 1)$, as follows

$$
v_{\mathrm{C}}^{\mathrm{barr}}(x, v d \gg 1)=\frac{(N-1)\left(\sqrt{2 I_{L}}+\sqrt{2 I_{R}}\right)^{2} \rho_{L}(x) \rho_{R}(x)}{2\left[\rho_{L}(x)+(N-1) \rho_{R}(x)\right]^{2}},
$$

and

$$
v_{\mathrm{C}}^{\mathrm{step}}(x, v d \gg 1)=\frac{I_{L} \rho_{L}(x)+(N-1) I_{R} \rho_{R}(x)}{\rho_{L}(x)+(N-1) \rho_{R}(x)} .
$$

Replacing the expressions for $\rho_{L}(x)$ and $\rho_{R}(x)$ in the equations above, one obtains after some manipulation

$$
v_{\mathrm{C}}^{\mathrm{barr}}(x, v d \gg 1)=\frac{\left(\sqrt{2 I_{L}}+\sqrt{2 I_{R}}\right)^{2} / 8}{\cosh ^{2}\left[\left(\sqrt{2 I_{L}}+\sqrt{2 I_{R}}\right)\left(x+x_{0}\right)\right]},
$$

while

$$
v_{\mathrm{C}}^{\text {step }}(x, v d \gg 1)=\frac{I_{L}-I_{R}}{1+\exp \left\{2\left[\left(\sqrt{2 I_{L}}+\sqrt{2 I_{R}}\right)\left(x+x_{0}\right)\right]\right\}},
$$

where

$$
\begin{aligned}
x_{0}(N) & =\frac{1}{2\left(\sqrt{I_{L}}+\sqrt{I_{R}}\right)} \\
& \times\left[d\left(\sqrt{I_{L}}-\sqrt{I_{R}}\right)+\frac{1}{\sqrt{2}} \ln \left(\frac{\sqrt{I_{R}}(N-1)}{\sqrt{I_{L}}}\right)\right] .
\end{aligned}
$$

Note that the dependence on $N$ is hidden now in the parameter $x_{0}(N)$, that signals the location of the peak feature. For $N=2$, these equations reduce to the ones found in Ref. [15]. These equations are only valid for $-d / 2 \leq x \leq d / 2$, and $I_{L}>I_{R}$. For $I_{L}=I_{R}$ and $N=2$, $x_{0}(2)=0$ and $v_{\mathrm{C}}^{\text {step }}(x, v d \gg 1) \equiv 0$; only in this case Eq. 23 coincides with the homonuclear result of Eq. (17) for $v_{\mathrm{C}}^{\mathrm{barr}}(x, v d \gg 1)$. For any other $N$ between 1 and $2, v_{\mathrm{C}}^{\text {step }}(x, v d \gg 1)$ still vanishes for $I_{L}=I_{R}$, but the correct homonuclear limit for $v_{\mathrm{C}}^{\mathrm{barr}}(x, v d \gg 1)$ as given by Eq. 17) cannot be obtained as a limit from Eq. 23. This is quite reasonable, as the starting point for the derivation of Eqs. 23 24 above is that in the dissociation limit the density can be written as $\rho_{N}^{>}(x)=\rho_{L}(x)+(N-$ 1) $\rho_{R}(x)$, with $\rho_{L}(x), \rho_{R}(x)$ being the densities of the left and right wells, or viceversa. And such simplification for the interacting density is not possible (except for $N=2$ ), as can be seen from Eq. (19). The explanation for this apparently paradoxical situation is given below.

The structure "step" + "barrier/shoulder" observed in Fig. 4 has been already discussed previously [13, 15], but its dependence on $N$, to the best of our knowledge has been not studied before. Using the parameters corresponding to Fig. 4, one obtains that $x_{0}(1.2) \simeq$ $1.24, x_{0}(1.4) \simeq 1.49, x_{0}(1.6) \simeq 1.63, x_{0}(1.8) \simeq 1.74$, $x_{0}(2) \simeq 1.82$, in agreement with the position of the peak in the shoulder, which is located at $x=-x_{0}(N)$. Also, $I_{L}-I_{R}=\left(\alpha_{L}^{2}-\alpha_{R}^{2}\right) / 2 \simeq 0.42$.

The physics behind the barrier/shoulder and step features in $\Delta v_{\mathrm{KS}}^{>}(x)$ have been already discussed for $N=2$, and here we provide additional insight by considering the case of fractional $N$. The crucial concept is that the effective single-particle potential $\Delta v_{\mathrm{KS}}^{>}(x)$ should manage to reproduce the interacting ground-state density, as displayed in Fig. 2. The step structure forms around 
the "atom" with the higher ionization potential (the left atom in Fig. 2), and locally induces the "equilibrium" condition $\varepsilon_{L}+I_{L}-I_{R}=\varepsilon_{R}$, which allows the exclusive population of the right-well in Fig. 2, as soon as $N>1$. The barrier/shoulder structure comes from $v_{\mathrm{C}}^{\mathrm{barr}}(x)$, and as in the homonuclear case its role is provide a barrier that induces the decoupling of the two atoms, in the dissociation limit. This can be seen clearly from Eq.(21): $v_{\mathrm{C}}^{\mathrm{barr}}(x, v d \gg 1)$ will display its maximum strength at the coordinate $x$ where the left and right densities have its maximum overlap. in "units" of the density $\rho_{N}(x)$. By inspection of Eq. 21] is easy to check that this happens when $\rho_{L}(x)=(N-1) \rho_{R}(x)$. Solving this equation for $x$, one obtains Eq. 225 in an alternative way. As $N$ increases beyond 1 , the effective right-related density $(N-1) \rho_{R}(x)$, and the point of maximum overlap between the two atomic density distributions moves closer to the atom with the higher ionization energy, as seen in Fig. 4. These considerations also explain why the shoulder/barrier peak moves closer to the left well as $N$ increases in Fig. 4.

Proceeding analogously, the following expressions are obtained for $x \leq-d / 2$,

$$
v_{\mathrm{C}}^{\mathrm{barr}}(x, v d \gg 1)=\frac{\left(\sqrt{2 I_{L}}-\sqrt{2 I_{R}}\right)^{2} / 8}{\cosh ^{2}\left[\left(\sqrt{2 I_{L}}-\sqrt{2 I_{R}}\right)\left(x+x_{0}^{\prime}\right)\right]},
$$

$v_{\mathrm{C}}^{\text {step }}(x, v d \gg 1)=\frac{I_{L}-I_{R}}{1+\exp \left\{2\left[\left(\sqrt{2 I_{L}}-\sqrt{2 I_{R}}\right)\left(x+x_{0}^{\prime}\right)\right]\right\}}$,

with

$$
\begin{aligned}
x_{0}^{\prime}(N) & =\frac{1}{2\left(\sqrt{I_{L}}-\sqrt{I_{R}}\right)} \\
& \times\left[d\left(\sqrt{I_{L}}+\sqrt{I_{R}}\right)-\frac{1}{\sqrt{2}} \ln \left(\frac{\sqrt{I_{R}}(N-1)}{\sqrt{I_{L}}}\right)\right] .
\end{aligned}
$$

Using once more the parameters of Fig. 4, one obtains $x_{0}^{\prime}(1.2) \sim 12.62, x_{0}^{\prime}(2) \sim 12.43 . \quad$ As before, $v_{\mathrm{C}}^{\text {barr }}(x, v d \gg 1)$ presents a maximum at $x=-x_{0}^{\prime}(N)$, while $v_{\mathrm{C}}^{\text {step }}(x, v d \gg 1)$ vanishes exponentially for $x<$ $-x_{0}^{\prime}(N)$, and increases monotonically towards its limiting value $I_{L}-I_{R}$ for $x>-x_{0}^{\prime}(N)$. Interestingly, the sum of both potentials also increases monotonically around $-x_{0}^{\prime}(N)$.

We display in Fig. 5 how the sum of the barrier and step contributions to $\Delta v_{\mathrm{KS}}^{>}(x)$ combines to yield the shoulder/barrier feature centered at $x=-x_{0}(1.4) \simeq$ -0.39 . We remark that this shoulder/barrier structure in the exact KS potential at the dissociation limit appears exactly at the position of the maximum overlap of the left and (effective) right density distributions, centered in this case at $x=-5$ and $x=5$, respectively. The lower panel gives a global view of $\Delta v_{\mathrm{KS}}^{>}(x)$, showing the marked asymmetry of $v_{\mathrm{C}}^{\text {step }}(x)$, that although associated with the electron located at the left-well at $x=-5$,

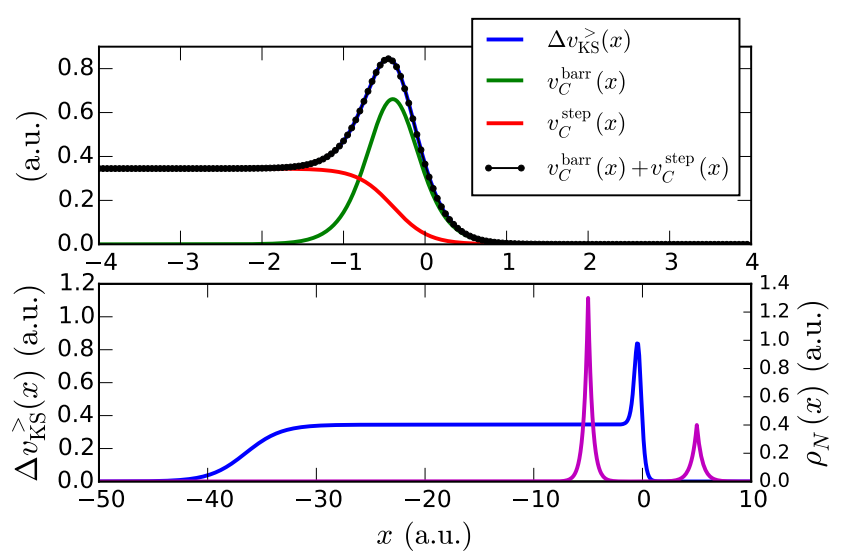

FIG. 5. $\Delta v_{\mathrm{KS}}^{>}(x)$ for $v=1, \lambda=1.3, N=1.4$, and $d=10$. Top panel: detail of the shoulder/barrier contribution $v_{\mathrm{C}}^{\text {barr }}(x)$ and the step contribution $v_{\mathrm{C}}^{\text {step }}(x)$; lower panel: global view of $\Delta v_{\mathrm{KS}}^{>}(x)$, and the associated density centered about $\pm d / 2=$ \pm 5 . For these parameters, the plateau region has the height $I_{L}-I_{R}=0.345$.

extends much further towards the left side than towards the right side, taking as reference the left-well coordinate. From Eq. (28), one obtains that $x_{0}^{\prime}(1.4) \simeq 40$, in good agreement with the beginning of the left side of $\Delta v_{\mathrm{KS}}^{>}(x)$. The length of the plateau or step in $v_{\mathrm{C}}^{\text {step }}(x)$ may be estimated from the difference $x_{0}^{\prime}(N)-x_{0}(N)$ : it increases linearly with $d$, while display a logarithmic dependence on $N$, decreasing its length as $N$ increases beyond 1 .

A two-electron one-dimensional model of a heterodiatomic molecule composed of two-open shell atoms has been also considered [25, with the Coulomb interaction being replaced by a soft-Coulomb potential. Their numerical results, restricted to the case $N=2$, are similar to ours, as they also obtained the exact KS potential with a shoulder/barrier and step features.

\section{Further discussions}

\section{The dissociation of the $\mathrm{H}_{2}^{+}$molecule}

For $\lambda=1$ (homonuclear molecule), we have used as definition of the dissociation limit the condition $e^{-2 v d} \ll$ 1. For $\lambda \neq 1$ (heteronuclear molecule), on the other side, the condition for dissociation modifies to $2 \sqrt{\lambda} e^{-v d} \ll$ $|\lambda-1|$. This suggest that for $\lambda \rightarrow 1$, for increasing $d$ the molecule may transition from a quasi-homonuclear configuration to a quasi-heteronuclear configuration; the situation is particularly interesting for the case of $N \rightarrow 1^{+}$. The critical value of $d$ for that transition is approximately given by the equation $e^{-v d^{*}}=|\lambda-1| /(2 \sqrt{\lambda})$. Solving for $d^{*}$, it yields

$$
d^{*} \simeq-\frac{1}{v} \ln \left(\frac{|\lambda-1|}{2 \sqrt{\lambda}}\right)
$$


If $\lambda \rightarrow 1$ from above or from below, $d^{*} \rightarrow \infty$ and the molecule is in the homonuclear limit for any finite value of $d$. Writing $\lambda=1+\epsilon$, with $\epsilon \ll 1, d^{*}(\lambda=1+\epsilon) \sim$ $-\ln |\epsilon| / v ;$ for $d \lesssim d^{*}$, the molecule (a one-dimensional mimic of the $H_{2}^{+}$molecule if $N \rightarrow 1^{+}$) will display a density distribution essentially of the homonuclear case, which on the other side is expected since $\lambda \sim 1$. But for $d \gtrsim d^{*}$, the density will start to display instead a quasi-heteronuclear configuration, with electrons being transferred from the atom with the lower ionization potential towards the one with the higher ionization potential. This is not as intuitive as in the previous case, since in principle $\lambda \sim 1$ is associated with a "bonding" charge distribution. This explains why the homonuclear limit of Eq. 23) does not coincide with the strict homonuclear result of Eq. (17): for arriving to the former we have assumed that the system is in a heteronuclear configuration such that $d \gtrsim d^{*}$, and after this is not possible to recover the homonuclear limit with $d \lesssim d^{*}$. The results displayed in Figs. 4 and 5 are well inside the heteronuclear regime, considering that $d^{*} \simeq 1.86$ and $d^{*} \simeq 2.03$ in these two figures, respectively.

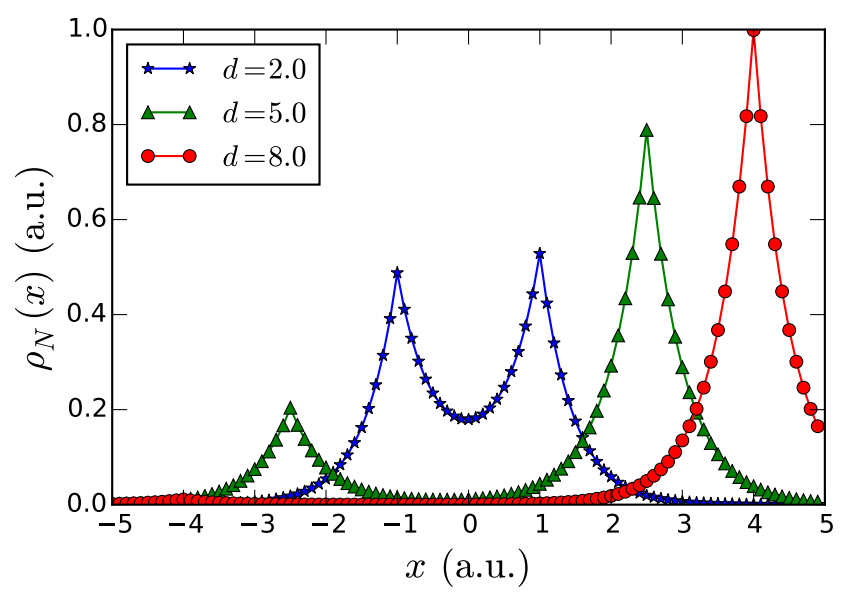

FIG. 6. Interacting density versus coordinate $x$, for different molecule's sizes. $v=1, \lambda=0.99$, and $N=1.01$. The configuration transition homonuclear $\rightarrow$ heteronuclear is evident as $d$ passes through $d^{*} \sim 5.3$.

We display in Fig. 6 an example of this situation. For these parameters, $d^{*} \sim 5.3$ from Eq. 229 . Note that this dramatic change in the molecule's electronic density from a $H^{+0.5} \cdots H^{+0.5}$-like configuration to a $H^{+1} \cdots H$-like configuration is achieved through the "step" contribution to $\Delta v_{\mathrm{KS}}^{>}(x)$, whose height $I_{R}-I_{L} \simeq v\left(1-\lambda^{2}\right) \simeq 0.02$ is very small, and can be as small as desired by choosing $\lambda$ closer to 1 .

The limit $N \rightarrow 1^{+}$of these results suggest a simple intuitive scenario for the "physical" dissociation process of the real tridimensional $H_{2}^{+}$molecule. As the separation between the two protons increases beyond the equilibrium distance, the molecule will start to feel increas- ingly the effect of any of the symmetry breaking fields from the environment (represented in our model by having $\lambda \neq 1$ ), and no matter how small this breaking field may be, charge will be transfered from one proton to the other about some critical distance $d^{*}$. After this, and by increasing $d$ further, the probability for the molecule to return to the symmetric charge distribution will become extremely small, as the tunneling probability for this process decreases exponentially as $d$ increases.

\section{The anomalous scaling of $v_{K S}(x)$ with the contact repulsion parameter $\gamma$}

It is also of some fundamental interest to analyze how the KS potential scales with $\gamma$. According to the ensemble generalization of the $\mathrm{KS}$ formulation of ground-state DFT [10,

$$
v_{\mathrm{KS}}^{N}(x)=v_{\mathrm{ext}}(x)+v_{\mathrm{HXC}}\left(\left[\rho_{N}\right] ; \gamma\right),
$$

with

$v_{\mathrm{HXC}}\left(\left[\rho_{N}\right] ; \gamma\right)=v_{\mathrm{H}}\left(\left[\rho_{N}\right] ; \gamma\right)+v_{\mathrm{X}}\left(\left[\rho_{N}\right] ; \gamma\right)+v_{\mathrm{C}}\left(\left[\rho_{N}\right] ; \gamma\right)$

$v_{\mathrm{H}}\left(\left[\rho_{N}\right] ; \gamma\right), v_{\mathrm{X}}\left(\left[\rho_{N}\right] ; \gamma\right), v_{\mathrm{C}}\left(\left[\rho_{N}\right] ; \gamma\right)$ are the Hartree, exchange, and correlation contributions to the KS potential, respectively. They depend on the contact parameter $\gamma$. For example, within our model

$$
v_{\mathrm{H}}\left(\left[\rho_{N}\right] ; \gamma\right)=\int d x^{\prime} \rho_{N}\left(x^{\prime}\right) \gamma \delta\left(x-x^{\prime}\right)=\gamma \rho_{N}(x)
$$

Besides, $v_{\mathrm{X}}\left(\left[\rho_{N}\right] ; \gamma\right)=-v_{\mathrm{H}}\left(\left[\rho_{N}\right] ; \gamma\right)$ for $0 \leq N \leq 1$, while 20.

$v_{\mathrm{X}}\left(\left[\rho_{N}\right] ; \gamma\right)=-\frac{N^{2}-2 N+2}{N^{2}} v_{\mathrm{H}}\left(\left[\rho_{N}\right] ; \gamma\right)+\frac{2(2-N)}{N^{3}} U\left[\rho_{N}\right]$

for $1<N \leq 2$. Here,

$$
\begin{aligned}
U\left[\rho_{N}\right] & =\frac{1}{2} \int d x \int d x^{\prime} \rho_{N}^{>}(x) \rho_{N}^{>}\left(x^{\prime}\right) \gamma \delta\left(x-x^{\prime}\right) \\
& =\frac{\gamma}{2} \int d x\left[\rho_{N}^{>}(x)\right]^{2} .
\end{aligned}
$$

The problem here is that for $\gamma \rightarrow \infty$ (our case), $v_{\mathrm{H}}\left(\left[\rho_{N}\right] ; \gamma\right), v_{\mathrm{X}}\left(\left[\rho_{N}\right] ; \gamma\right)$, and $v_{\mathrm{C}}\left(\left[\rho_{N}\right] ; \gamma\right)$ all diverge linearly with $\gamma$. On the other side, our previous results are such that both $\Delta v_{\mathrm{KS}}^{<}(x)$ and $\Delta v_{\mathrm{KS}}^{>}(x)$ are finite. How is this possible?.

The answer is clear for $0 \leq N \leq 1$ : here $v_{\mathrm{H}}\left(\left[\rho_{N}\right] ; \gamma\right)+$ $v_{\mathrm{X}}\left(\left[\rho_{N}\right] ; \gamma\right) \equiv 0$, canceling mutually, whatever the value of $\gamma \cdot v_{\mathrm{C}}\left(\left[\rho_{N}\right] ; \gamma\right) \equiv 0$ also, since for $N \leq 1$ the system is not correlated. This is of course consistent with the result $\Delta v_{\mathrm{KS}}^{<}(x) \equiv 0$ obtained before. The situation for $1<N \leq 2$ is more interesting, since in this case the cancellation of the divergent contributions in $v_{\mathrm{H}}\left(\left[\rho_{N}\right] ; \gamma\right)$ and $v_{\mathrm{X}}\left(\left[\rho_{N}\right] ; \gamma\right)$ is only partial. For $v_{\mathrm{HXC}}\left(\left[\rho_{N}\right] ; \gamma\right)$ in Eq. 30 
to remain finite, one concludes that a necessary condition is that $v_{\mathrm{C}}\left(\left[\rho_{N}\right] ; \gamma\right)$ must be the sum of two contributions: $v_{\mathrm{C}}\left(\left[\rho_{N}\right] ; \gamma\right)=\tilde{v}_{\mathrm{C}}\left(\left[\rho_{N}\right] ; \gamma\right)+\Delta v_{\mathrm{KS}}^{>}(x) . \quad \tilde{v}_{\mathrm{C}}\left(\left[\rho_{N}\right] ; \gamma\right)$ must scale linearly with $\gamma$, and should fulfill the cancellation constraint $v_{\mathrm{H}}\left(\left[\rho_{N}\right] ; \gamma\right)+v_{\mathrm{X}}\left(\left[\rho_{N}\right] ; \gamma\right)+\tilde{v}_{\mathrm{C}}\left(\left[\rho_{N}\right] ; \gamma\right) \equiv 0$, while the second contribution, which remains finite even when $\gamma \rightarrow \infty$ is what we have denoted as $\Delta v_{\mathrm{KS}}^{>}(x)$ in Eq. 16. In the more general case of finite $\gamma$ or by using more realistic (Coulomb-like) potentials, we expect that both $v_{\mathrm{H}}\left(\left[\rho_{N}\right] ; \gamma\right)+v_{\mathrm{X}}\left(\left[\rho_{N}\right] ; \gamma\right)+\tilde{v}_{\mathrm{C}}\left(\left[\rho_{N}\right] ; \gamma\right)$ and $\Delta v_{\mathrm{KS}}^{>}(x)$ will be different from zero [26. Our strongly interacting model exhibits somehow this anomalous scaling in its extreme limit, forcing the complete mutual cancellation of all the "normal" scaling contributions to $v_{\mathrm{HXC}}\left(\left[\rho_{N}\right] ; \gamma\right)$, and keeping finite only the "anomalous" contribution $\Delta v_{\mathrm{KS}}^{>}(x)$, that is of zero-order in $\gamma$.

We expect then that the main result of this section regarding the anomalous scaling property of the KS potential to remain valid even after the replacement of the delta-function interaction potential by more realistic models [26, 27.

The fact that the Kohn-Sham potential must include a term with an anomalous scaling in the strength of the Coulomb interaction has been recently noticed [27], and our present results confirm even more clearly this important point. The conclusion is clear: without these anomalous scaling terms, the dissociation limit of homonuclear and heteronuclear molecules will be poorly described, since the KS potential will suffer from the absence of the barrier and step features in it. As discussed in Ref. [27], the absence of these "Mott barriers" has also important consequences on the electronic properties of strongly correlated solids.

\section{CONCLUSIONS}

Some unusual features of the exact Kohn-Sham potential for finite systems with a fractional number of elec- trons have been discussed. The exact ground-state density of a strongly interacting model for a one-dimensional diatomic molecule has been obtained, from which by reverse engineering the Kohn-Sham potential is derived for all fractional molecules occupancies between 0 and 2. Large differences exist between the results for the homonuclear and heteronuclear cases, particularly in the dissociation limit. For the homonuclear case, and in the dissociation limit, the exact Kohn-Sham potential builds a barrier at the midpoint between the two atoms, whose strength increases linearly with $N$, with $1<N \leq 2$. In the heteronuclear case, the KS potential exhibits a peak related to the barrier of the homonuclear case, exactly centered at the coordinate of maximum overlap between the left and right density distributions. Besides, it also displays a plateau around the atom with the higher ionization potential, whose size (but not its strength) depends on $N$. An anomalous zero-order scaling of the KS potential with regards to the strength of the electronelectron repulsion is clearly displayed by our model, without which both the unusual barrier and plateau features will be absent.

\section{ACKNOWLEDGMENTS}

The authors acknowledge J. Fuhr and G. Bocan for useful discussions, and to J. Luzuriaga for a critical reading of the manuscript. This work was sponsored by PICT 2012-0379 of the ANPCyT, Argentina. C.R.P. is partially supported by CONICET.
[1] R. G. Parr and W. Yang, in Density Functional Theory of Atoms and Molecules. (Oxford University Press, New York, 1989).

[2] R. M. Dreizler and E. K. U. Gross, in Density Functional Theory. (Springer-Verlag, Berlin, 1990).

[3] W. Kohn and L. Sham, Phys. Rev. 140, A1135 (1965).

[4] J. P. Perdew, A. Ruzsinszky, G. I. Csonka, O. A. Vydrov, G. E. Scuseria, V. N. Staroverov, and J. Tao, Phys. Rev. A 76, 040501(R) (2007).

[5] A. Ruzsinsky, J. P. Perdew, G. I. Csonka, O. A. Vydrov, and G. E. Scuseria, J. Chem. Phys. 126, 104102 (2007).

[6] P. Mori-Sánchez, A. J. Cohen, and W. Yang, J. Chem. Phys. 125, 201102 (2006).

[7] P. Mori-Sánchez, A. J. Cohen, and W. Yang, Phys. Rev. Lett. 100, 146401 (2008).

[8] D. J. Tozer, J. Chem. Phys. 119, 12697 (2003).
[9] J. P. Perdew, V. N. Staroverov, J. Tao, and G. E. Scuseria, Phys. Rev. A 78, 052513 (2008)

[10] J. P. Perdew, R. G. Parr, M. Levy, and J. L. Balduz, Phys. Rev. Lett. 49, 1691 (1982).

[11] J. P. Perdew and M. Levy, Phys. Rev. Lett. 51, 1884 (1983).

[12] L. J. Sham and M. Schlüter, Phys. Rev. Lett. 51, 1888 (1983).

[13] J. P. Perdew. What do the Kohn-Sham orbitals mean? How do atoms dissociate? In Density Functional Methods in Physics; R. M. Dreizler and J. da Providence (eds), p. 265. (Plenum, New York, 1985).

[14] A. J. Cohen, P. Mori-Sánchez, and W. Yang, Chemical Reviews 112, 289 (2012).

[15] N. Helbig, I. V. Tokatly, and A. Rubio, J. Chem. Phys. 131, 224105 (2009). 
[16] See for example S. Gasiorowitz, Quantum Physics (Wiley, New York, 1996).

[17] V. I. Yukalov and M. D. Girardeau, Laser Phys. Lett. 2, 375 (2005). In a few words, the ground-state of the diatomic molecule for $N=2$ is a spin-singlet. Since the total wave-function must be antisymmetric under the exchange of the two electrons, this implies that the spacial part of it should be symmetric (like bosons). On the other side, for $\gamma \rightarrow \infty$ the contact potential imposes the vanishing condition $\Psi^{\gamma \rightarrow \infty}(x, x)=0$. Eq. (4) is then the exact expression for the spatial part of the ground-state $N=2$ electronic wavefunction that is both symmetric, and vanishes when the two electronic coordinates coincide.

[18] For Eq. (11) to be valid, the total energy must satisfy the "convexity" condition $E(N+1)+E(N-1)>2 E(N)$. Taking $N=1$, this implies the condition $E(2)=\varepsilon_{+}+$ $\varepsilon_{-}>2 E(1)=2 \varepsilon_{+}$, which is strictly satisfied, since $\varepsilon_{-}>$ $\varepsilon_{+}$.

[19] E. Sagvolden and J. P. Perdew, Phys. Rev. A 77, 012517 (2008).

[20] P. Gori-Giorgi and A. Savin, Int. J. Quantum Chem. 109, 2410 (2009).
[21] T. Gould and J. Toulouse, Phys. Rev. A 90, 050502(R) (2014).

[22] This is easily seen by using the starting equation for the reverse engineering procedure, Eq. 13): for $\rho_{N}^{>}(x) \sim$ $N\left[\rho_{L}(x)+\rho_{R}(x)\right]$, the factor $N$ cancels.

[23] M. A. Buijse, E. J. Baerends, and J. G. Snijders, Phys. Rev. A 40, 4190 (1989).

[24] O. V. Gritsenko and E. J. Baerends, Theor. Chem. Acc. 96, 44 (1997).

[25] D. G. Tempel, T. J. Martínez, and N. T. Maitra, J. Chem. Theory Comp. 5, 770 (2009).

[26] We have partially confirmed this by replacing the contact potential $\gamma \delta\left(x-x^{\prime}\right)$ by a "soft" one-dimensional Coulomb potential $1 /\left(\left|x-x^{\prime}\right|+a\right)$, with $a$ being a small positive constant. In the limit $a \ll 1$, we have checked: a) the nearly complete cancellation of the "normal" contributions to $v_{H x c}(x)$ for any $x$; and b) the existence of an anomalous contribution to $v_{H x c}(x)$, whose size does not depends on $a$, and that remains finite whatever the infinitesimal value of $a$ is.

[27] Z.-Y. Ying, V. Brosco, G. Lopez, D. Varsano, P. GoriGiorgi, and J. Lorenzana, (arXiv:1510.03425). 